Our Nature (2008)6: 58-66

\title{
Desmids from Bees-hazaar Lake, Chitwan, Nepal
}

\author{
S.K. Rai ${ }^{1}$, R.K. Rai ${ }^{2}$ and N. Pau del ${ }^{1}$ \\ ${ }^{1}$ Department of Botany, P.G. Campus, T.U., Biratnagar, Nepal \\ ${ }^{2}$ Department of Botany, M.MA.M. Campus, T.U., Biratnagar, Nepal
}

Received: 19.03.2008, Accepted: 04.07.2008

\begin{abstract}
A total 36 taxa belonging to 7 genera of desmids have been described and illustrated from Beeshazaar lake of which 11 taxa are recorded for the first time for Nepal. This is a preliminary work on the desmids from this lake.
\end{abstract}

Key words: Algae, Bees-hazaar Lake, Desmids, Nepal.

\section{Introduction}

Bees-hazaar lake (Latitude 27037'04.6"N, Longitude 84²6'11.3"E; altitude $286 \mathrm{~m}$ amsl; area ca $100 \mathrm{ha}$ ) is an oxbow lake (Ramsar site) situated in Tikauli-3, Gitanagar VDC, Chitwan, surrounded by Barandabhar Corridor Forest, an extension of the buffor zone of Chitwan National Park. The lake is connected with other six ponds, ghols and swamps towards its south-east end whereas it forms many finger-like projections towards the north. The rich in total nitrogen and orthophosphate and low in transparency level ranked the lake as hypereutrophic cat egory.

The work on desmids of Nepal has been carried out by Hirano (1955, 1963, 1969, 1984), Förster (1965), Kusel-fetzmann (1969), Hickel (1973), Ichimura and Kasai (1982), Shrestha and Manandhar (1983), Nakanishi (1986), Bando et al. (1989), Habib and Chat urvedi (1995, 1997), Rai (2007), and Rai and Misra (2008). The literature revealed that the desmids flora of Nepal has not been explored so far extensively and further more, the terai plain is least studied. Here, an attempt has been made to study the desmids from Bees-hazaar Lake.
In this paper, taxonomy and morphology of 36 taxa of desmids belonging to 7 genera (Closterium 4, Pleurotaenium 1, Euastrum 5, Micrasterias 5, Cosmarium 16, Arthrodesmus 1, Staurastrum 4) has been described and illustrated. Out of these, 11 taxa viz., Euastrum elegans (Bréb.) Kütz., Micrasterias mahabuleshwarensis Hobs. var. surculifera Legerh., M. tropica Nordst., var. polonica Eichl. et Gutw. f. evoluta Scott et Prescott, Cosmarium bioculatum Bréb., C. contractum Kirchn. var. pachydermum Scott et Prescott, $C$. cymatopleurum Nordst. var. subtropicum Islam, $C$. punctulatum Bréb. var. subpunctulatum (Nordst.) Borges, $C$. striolatum Näg., Arthrodesmus convergens Ehr. var. curtus Turn., Staurastrum ef bifidum Bréb. in Ralfs, and St. javanicum (Nordst.) Turn. var. apiculifenum (Turn.) Krieg. have been reported for the first time for Nepal. All the reports are new for the lake as no work has been done hitherto.

\section{Mate rials and meth ods}

Algal samples were collected from five 
S.K.Rai, R.K. Rai and N. Paudel / Our Nature (2008) 6: 58-66

different sites of Bees-hazaar lake during the period of June to September, 2007. All the samples were taken by squeezing out the roots of Eichhornia crassipes, Pistia stratiotes and submerged aquatic macrophytes. Samples were tagged and labeled then preserved in $4 \%$ formaldehyde solution on the spot. Morpho-taxonomy of the desmids was studied in the Laboratory of Department of Botany, P.G. Campus by screening and camera-lucida drawings. Identification was done on the basis of illustration and dimension of the relevant literat ure and monographs mentioned below each taxon's name in the text.

Explanation of symbols and abbreviations

L: Cell length

W: Cell diameter

MW: Median diameter

AW: Apical diameter

PW:Polar diameter

I: Isthmus diameter

$\mathrm{CN}$ : Sample number

D: Collection date

\section{Taxonomic de scriptions}

1. Closterium acerosum (Schr.) Ehr. ex Ralfs (P1. 1, Fig. 17)

Scott, A.M. and G.W. Prescott 1961, P. 9, P1. 3, Fig. 1

L $474 \mu \mathrm{m}$, MW $42 \mu \mathrm{m}$, AW 4.5-6 $\mu \mathrm{m}$; CN BH-18; D 27.8.2007

Distribution: Pangka, $4600 \mathrm{~m}$; Longponga, 4650 m; Dudhpokhari, $4750 \mathrm{~m}$ (Watanabe, 1982); Mahendranagar (Habib and Chaturvedi, 1995; 1997); Koshi Tappu, 206 m (Rai and Misra, 2008)

2. Closterium dianae Ehr. ex Ralfs var. dianae (P1. 1, Fig. 14)

Flint, E.A. and D.B. Williamson 1998, P. $75, \mathrm{Pl}$. 2, Fig. 7
L $203 \mu \mathrm{m}$, MW $17 \mu \mathrm{m}$, AW 2-3 $\mu \mathrm{m}$; CN BH-4; D 25.6.2007

Distribution: Begnas lake, $960 \mathrm{~m}$; Hetauda, $500 \mathrm{~m}$ (Wat anabe, 1982); Koshi T appu, 206 m (Rai and Misra, 2008)

3. Closterium ehrenbergii Menegh. ex Ralfs (P1. 1, Fig. 15)

Nurul Islam, A.K.M. 1970, P. 910, Pl. 6, Fig. 14

L $374 \mu \mathrm{m}$, MW $64 \mu \mathrm{m}$, AW 8-10 $\mu \mathrm{m}$; CN BH-5; D 25.6.2007

Distribution: Mugling, 500 m; Hetauda, 500 m (Wat anabe, 1982); Baudha, Pashupatinath, Bansbari in Kathmandu; Dulari in Jhapa; Itahari; Birganj (Ichimura and Kasai, 1982); Chabahil in Kathmandu (Bando et al., 1989); Mahendranagar (Habib and Chat urvedi, 1997); Koshi Tappu, 206 m (Rai and Misra, 2008)

4. Closterium rostratum Ehr. ex Ralfs (P1. 1, Fig. 18)

Kouwets, F.A.C. 1987, P. 207, P1. 5, Figs. 4-5

L $295 \mu \mathrm{m}$, MW $17 \mu \mathrm{m}$, AW 3-4 $\mu \mathrm{m}$; CN BH- 13; D 24.7.2007

Distribution: Dole, $4100 \mathrm{~m}$, Solu; Hetauda, $500 \mathrm{~m}$ (Wat anabe, 1982); Mahendranagar (Habib and Chaturvedi, 1997)

\section{Pleurotaenium baculoides (Roy et} Biss.) Playf. (P1. 1, Fig. 19)

Scott, A.M. and G.W. Prescott 1961, P. 14, P1. 3, Fig. 5

L $370 \mu \mathrm{m}$, MW $30 \mu \mathrm{m}$, AW 18-20 $\mu \mathrm{m}$, I 20 $\mu \mathrm{m} ; \mathrm{CN}$ BH-21; D 25.9.2007

Distribution: T audaha, 1350 m, Kathmandu (Bando et al., 1989)

6. Euastrum ansatum Ralfs var. dideltiforme Ducell. (P1. 2, Fig. 9) 
S.K.Rai, R.K. Rai and N. Paudel / Our Nature (2008) 6: 58-66

Nurul Islam, A.K.M. 1970, P. 916, P1. 16, 11. Micrasterias foliacea Bail. (P1. 1, Fig. Fig. 6

13)

L $85.8 \mu \mathrm{m}$, W $45 \mu \mathrm{m}$, PW $21 \mu \mathrm{m}$, I 11.6 Scott, A.M. and G.W. Prescott 1961, P. 46, $\mu \mathrm{m}$; CN BH-10; D 25.6.2007

P1. 20, Fig. 4

Distribution: Mewa valley (Hirano, 1984); L $70 \mu \mathrm{m}$, W $75 \mu \mathrm{m}$, I $17 \mu \mathrm{m}$; CN BH-1; D Maipokhari lake, 2150 m, Ilam (Rai, 2008)

25.6.2007

Distribution: Koshi Tappu, 206 m (Rai and

7. Euastrum bidentatum Näg. (Pl. 2, Fig. Misra, 2008)

14)

Capdevielle, P. and A. Coute' 1980, P. 880,

Pl. 2, Fig. 20

L $48 \mu \mathrm{m}$, W $30.5 \mu \mathrm{m}$, PW $18 \mu \mathrm{m}$, I $10 \mu \mathrm{m}$;

CN BH-10; D 25.6.2007

Distribution: Pheriche, $4200 \mathrm{~m}$, Khumbu

(Förster, 1965 as var. speciosum); Koshi

Tappu, 206 m (Rai and Misra, 2008)

12. Micrasterias mahabuleshwarensis

Hobs. var. surculifera Lagerh. (Pl. 2, Fig. 6)

Scott, A.M. and G.W. Prescott 1961, P. 50,

Pl. 16, Figs. 1-2

L $154 \mu \mathrm{m}$, W $134 \mu \mathrm{m}$, I $24 \mu \mathrm{m}$; CN BH-19;

D 27.8.2007

Distribution: New record for Nepal.

8. Euastrum elegans (Bréb.) Kütz. (Pl. 1, Fig. 3)

13. Micrasterias pinnatifida (Kütz.) Ralfs (P1. 2, Fig. 8)

Kouwets, F.A.C. 1987, P. 215, Pl. 8, Figs. Nurul Islam, A.K.M. 1970, P. 920, Pl. 10, 7-8

L $36 \mu \mathrm{m}$, W $24 \mu \mathrm{m}$, PW $16 \mu \mathrm{m}$, I $6 \mu \mathrm{m}$; CN

BH-8; D 25.6.2007

Figs. 3-7

L $54 \mu \mathrm{m}$, W $62 \mu \mathrm{m}$, I $10 \mu \mathrm{m}$; CN BH-11; D 24.7.2007

Distribution: New record for Nepal.

Distribution: Luitel Bhanjyang, $770 \mathrm{~m}$, Gorkha (Hirano 1955); Koshi T appa, 206 m

9. Euastrum platycerum Reinsch (P1. 1, (Rai and Misra, 2008)

Fig. 10)

Scott, A.M. and G.W. Prescott 1961, P. 33, Pl. 60, Fig. 4

14. Micrasterias radians Turn. (P1. 2, Fig. 7)

L $41 \mu \mathrm{m}$, W $35 \mu \mathrm{m}$, PW $12.5 \mu \mathrm{m}$, I $10 \mu \mathrm{m}$; CN BH-17; D 27.8.2007

Scott, A.M. and G.W. Prescott 1961, P. 51, Pl. 23, Fig. 1

Distribution: Koshi Tappu, 206 m (Rai and Misra, 2008)

L $125 \mu \mathrm{m}$, W $115 \mu \mathrm{m}$, I $21 \mu \mathrm{m}$; CN BH-6; D 25.6.2007

Distribution: T ahachal, 1300 m, Kathmandu

10. Euastrum spinulosum DelP. (P1. 1, Fig. (Hirano, 1955)

8)

Scott, A.M. and G.W. Prescott 1961, P. 40, Pl. 10, Fig. 3

L $45 \mu \mathrm{m}$, W $36 \mu \mathrm{m}$, PW $15 \mu \mathrm{m}$, I $11 \mu \mathrm{m}$;

CN BH-13; D 24.7.2007

Distribution: Koshi Tappu, 206 m (Rai and Misra, 2008)

15. Micrasterias tropica Nordst. var. polonica Eichl. et Gutw. f. evoluta Scott et Prescott (P1. 2, Fig. 5)

Scott, A.M. and G.W. Prescott 1961, P. 53, Pl. 16, Fig. 7

L $133 \mu \mathrm{m}$, W $103 \mu \mathrm{m}$, I $17.5 \mu \mathrm{m}$; CN BH19; D 27.8.2007 
S.K.Rai, R.K. Rai and N. Paudel / Our Nature (2008) 6: 58-66

Distribution: New record for Nepal.

L $24 \mu \mathrm{m}, \mathrm{W} 16.5 \mu \mathrm{m}, \mathrm{I} 4 \mu \mathrm{m}$; CN BH-19; D 27.8.2007

16. Cosmarium bioculatum Bréb. (Pl. 1, Distribution: Pheriche, $4200 \mathrm{~m}$, Khumbu Fig. 4)

Bharati, S.G. and G.R. Hegde 1982, P. 736, Pl. 11, Fig. 12

L $19 \mu \mathrm{m}$, W $19 \mu \mathrm{m}$, I $5 \mu \mathrm{m}$; CN BH-1; D 25.6.2007

Distribution: New record for Nepal.

(Förster, 1965)

21. Cosmarium javanicum Nordst. (P1. 1, Fig. 6)

Nurul Islam, A.K.M. and A.K. Yusuf Haroon 1980, P. 576, Pl. 11, Figs. 156-157; Pl. 13, Fig. 179

17. Cosmarium contractum Kirchn. var. L $155 \mu \mathrm{m}$, W $70 \mu \mathrm{m}$, I $32.5 \mu \mathrm{m}$; CN BH-1; pachydermum Scott et Prescott (P1. 1, Fig. D 25.6.2007

2)

Distribution: Luitel Bhanjyang, $770 \mathrm{~m}$, Scott, A.M. and G.W. Prescott 1961, P. 56, P1. 27, Fig. 6

L $34 \mu \mathrm{m}$, W $27 \mu \mathrm{m}$, I $6 \mu \mathrm{m}$; CN BH-12; D 24.7.2007

Distribution: New record for Nepal.

Gorkha (Hirano, 1955); T audaha, $1350 \mathrm{~m}$,

Kathmandu (Bando et al. 1989);

Mahendranagar (Habib and Chaturvedi, 1995); Koshi Tappu, 206 m (Rai and Misra, 2008)

18. Cosmarium cymatopleurum Nordst. var. subtropicum Islam (P1. 1, Fig. 5)

22. Cosmarium lundellii DelP. var. circulare (Reinsch) Krieg. (Pl. 2, Fig. 4)

Nurul Islam, A.K.M. 1970, P. 923, P1. 11, Bharati, S.G. and G.R. Hegde 1982, P. 744, Fig. 11

Pl. 1, Fig. 2

L $42 \mu \mathrm{m}$, W $29 \mu \mathrm{m}$, I $8.5 \mu \mathrm{m}$; CN BH-8; D 25.6.2007

L $55 \mu \mathrm{m}$, W $42 \mu \mathrm{m}$, I $16 \mu \mathrm{m}$; CN BH-13; D 24.7.2007

Remark: Present specimen has small dimension than the type.

19. Cosmarium granatum Bréb. (Pl. 1, Fig. 7)

Distribution: Mewa valley (Hirano, 1984);

Mahendranagar (Habib and Chaturvedi,

Tiffany, L.H. and M.E. Britton 1952, P. 1997)

186, P1. 51, Fig. 565

L $33 \mu \mathrm{m}$, W $24 \mu \mathrm{m}$, I $6 \mu \mathrm{m}$; CN BH-12; D 24.7.2007

Distribution: Luitel Bhanjyang, $770 \mathrm{~m}$, Gorkha; Pisang, 3100 m, Manang; Tukucha Moor, $2600 \mathrm{~m}$, Mustang (Hirano, 1955; 1963); Mahendranagar (Habib and Chat urvedi, 1997)

23. Cosmarium lundellii DelP. var. ellipticum West et West f. minus Prescott (Pl. 1, Fig. 11)

Bharati, S.G. and G.R. Hegde 1982, P. 744, P1. 1, Fig. 6

L $43 \mu \mathrm{m}$, W $39 \mu \mathrm{m}$, I $15 \mu \mathrm{m}$; CN BH-12; D 27.7.2007

Distribution: Koshi Tappu, 206 m (Rai and

20. Cosmarium impressulum Elfv. f. Misra, 2008)

minus Turn. (Pl. 1, Fig. 9)

Bharati, S.G. and G.R. Hegde 1982, P. 742, P1. 11, Fig. 3

24. Cosmarium maculatiforme Schm. (Pl. 2, Fig. 12) 
S.K.Rai, R.K. Rai and N. Paudel / Our Nature (2008) 6:58-66

Nurul Islam, A.K.M. 1970, P. 924, Pl. 14, L $78 \mu \mathrm{m}$, W $44 \mu \mathrm{m}$, I $18 \mu \mathrm{m}$; CN BH-14; D Fig. 1 24.7.2007

L $113 \mu \mathrm{m}$, W $62 \mu \mathrm{m}, \mathrm{I} 40 \mu \mathrm{m} ; \mathrm{CN}$ BH-18; D 27.8.2007

Distribution: Karyolung, 4300-4400 m, Khumbu (Förster, 1965)

Distribution: Luitel Bhanjyang, $770 \mathrm{~m}$, Gorkha (Hirano, 1955 as var. maior)

29. Cosmarium regnellii Wille Fa. (Pl. 1, Fig. 1)

25. Cosmarium margaritatum (Lund.) Roy et Biss. var. sublatum (Nordst.) Krieg. (Pl. 2, Fig. 10)

Prasad, B.N. and P.K. Misra 1992, P. 180, Pl. 21, Fig. 25

L $11.5 \mu \mathrm{m}$, W $10 \mu \mathrm{m}$, I $2.7 \mu \mathrm{m}$; CN BH-19; Scott, A.M. and G.W. Prescott 1961, P. 63, D 27.8.2007

Pl. 29, Fig. 4

Distribution: Pheriche, $4200 \mathrm{~m}$, Khumbu L $70 \mu \mathrm{m}$, W $64 \mu \mathrm{m}$, I $21 \mu \mathrm{m}$; CN BH-23; D 25.9.2007

(Förster, 1965); Taudaha, $1350 \mathrm{~m}$, Kathmandu (Bando et al., 1989); Rara lake, Distribution: Ankhu Khola, 630 m, Gorkha (Hirano, 1955)

26. Cosmarium obsoletum (Hantz.) Reinsch (P1. 2, Fig. 2)

Kouwets, F.A.C. 1987, P. 226, P1. 11, Fig. 15

L $51 \mu \mathrm{m}$, W $55 \mu \mathrm{m}$, I $24 \mu \mathrm{m}$; CN BH-18; D 27.8.2007

3030 m, Mugu (Wat anabe, 1995)

30. Cosmarium striolatum Näg. (Pl. 2, Fig. 11)

Bharati, S.G. and G.R. Hegde 1982, P. 752 , Pl. 10, Fig. 4

L $108 \mu \mathrm{m}$, W $63 \mu \mathrm{m}$, I $51 \mu \mathrm{m}$; CN BH-4; D 25.6.2007

Distribution: New record for Nepal.

Distribution: Luitel Bhanjyang, $770 \mathrm{~m}$, Gorkha (Hirano, 1955); Koshi Tappu, 206 m (Rai and Misra, 2008)

31. Cosmarium sublateriundatum West et West (P1. 2, Fig.1)

Nurul Islam, A.K.M. and A.K. Yusuf

27. Cosmarium punctulatum Bréb. var. subpunctulatum (Nordst.) Borges (P1. 2, Fig. 3)

Haroon 1980, P. 580, P1. 22, Figs. 263-264

L $41 \mu \mathrm{m}$, W $39 \mu \mathrm{m}$, I $13 \mu \mathrm{m}$; CN BH-22; D 25.9.2007

Scott, A.M. and G.W. Prescott 1961, P. 67, Distribution: Tukucha Moor, $2600 \mathrm{~m}$, P1. 31, Fig. 8

Mustang (Hirano, 1955); Pashupatinath, L $28 \mu \mathrm{m}$, W $25 \mu \mathrm{m}$, I $9 \mu \mathrm{m}$; CN BH-7; D 1300 m (Bando et al., 1989)

25.6.2007

Distribution: New record for Nepal.

32. Arthrodesmus convergens Ehr. var. curtus Turn. (Pl. 1, Fig. 12)

28. Cosmarium quadratum Ralfs ex Ralfs var. willei (Schm.) Krieg. et Gerl. (P1. 2, Fig. 13)

Kouwets, F.A.C. 1987, P. 231, P1. 11, Figs. 8-10

Scott, A.M. and G.W. Prescott 1961, P. 74, Pl. 34, Fig. 5

L $57 \mu \mathrm{m}, \mathrm{W} 84 \mu \mathrm{m}$ (with spines), I $20 \mu \mathrm{m}$; CN BH-1; D 25.6.2007

Distribution: New record for Nepal. 
S.K.Rai, R.K. Rai and N. Paudel / Our Nature (2008) 6: 58-66
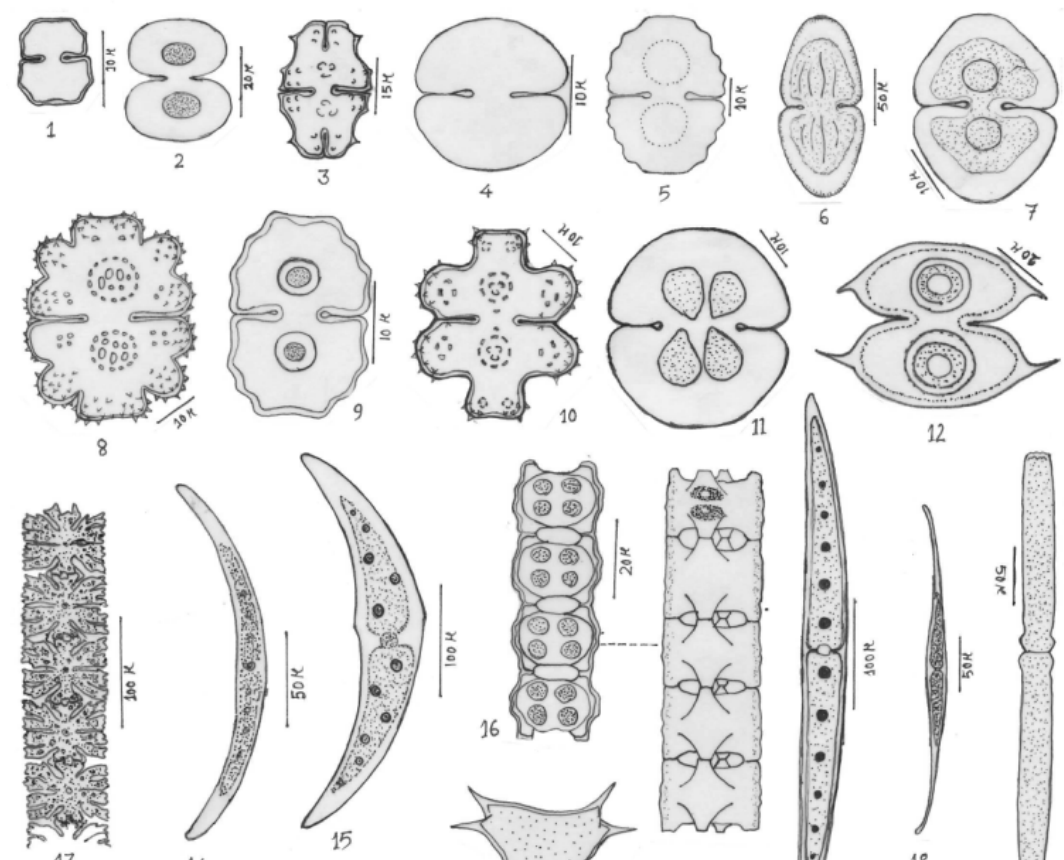

14

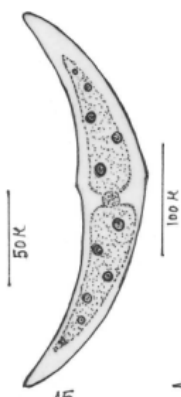

15
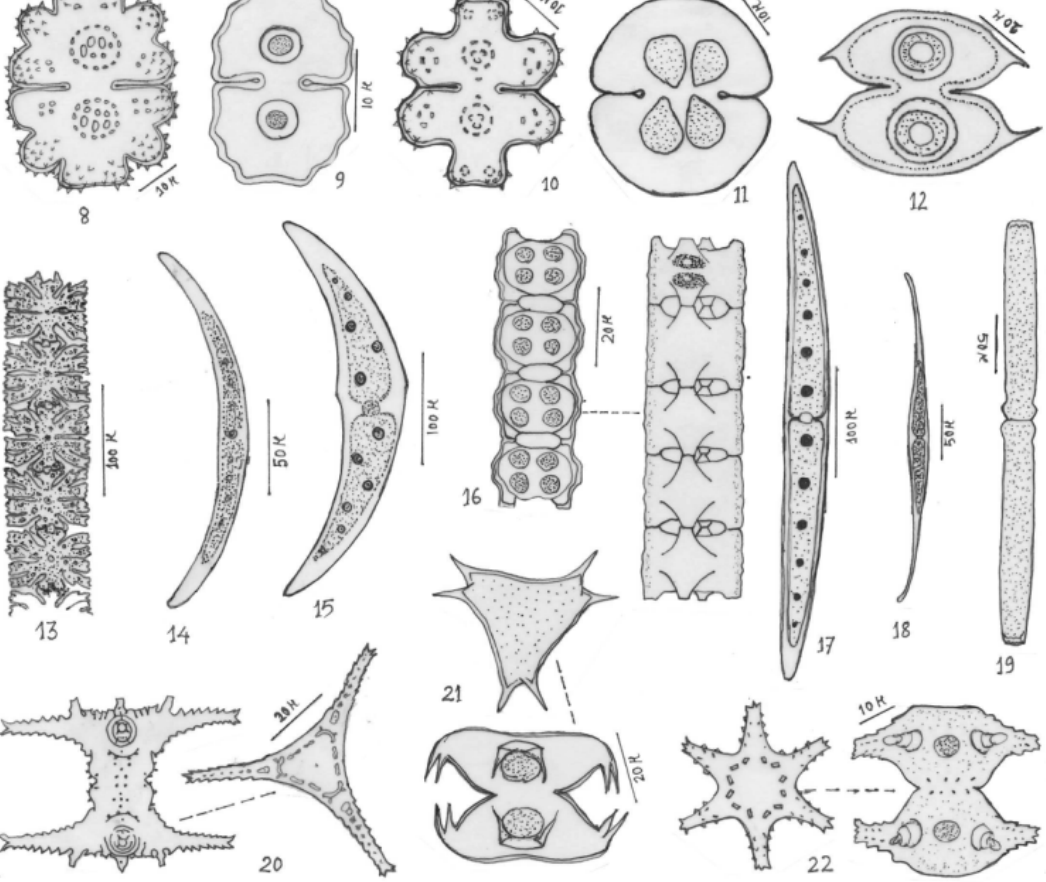

12

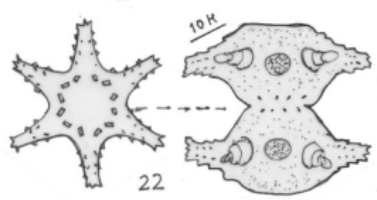

Plate 1

Fig.1 Cosmarium regnellii Wille Fa., Fig. 2 C. contractum Kirchn. var. pachydermum Scott et Prescott, Fig. 3 Euastrum elegans (Bréb.) Kütz., Fig. 4 Cosmarium bioculatum Bréb., Fig. 5 C. cymatopleurum Nordst. var. subtropicum Islam, Fig. 6 C. javanicum Nordst., Fig. 7 C. granatum Bréb., Fig. 8 Euastrum spinulosum DelP., Fig. 9 Cosmarium impressulum Elfv.f. minus Tum., Fig. 10 Euastrum platycerum Reinsch, Fig. 11 Cosmarium lundellii DelP. var. ellipticum West et West f. minus Prescott, Fig. 12 Arthrodesmus convergens Ehr. var. curtus Turn., Fig. 13 Micrasterias foliacea Bail., Fig. 14 Closterium dianae Ehr. ex Ralfs var. Diana, Fig. 15Cl. ehrenbergii Menegh. ex Ralfs, Fig. 16 Desmidium baileyi (Ralfs) Nordst. var. baileyi f. tetragonum Nordst., Fig. 17 Closterium acerosum (Schr.) Ehr. ex Ralfs, Fig. $18 \mathrm{Cl}$. rostratum Ehr. ex Ralfs, Fig. 19 Pleurotaenium baculoides (Roy et Biss.) Playf., Fig. 20 Staurastrumjavanicum (Nordst.) Turn. var. apiculiferum (Turn.) Krieg., Fig. 21 St. cf bifidum Bréb. in Ralfs, Fig. 22 St. sexcostatum Bréb. ex Ralfs var. productum (W. West) G.S. West. 

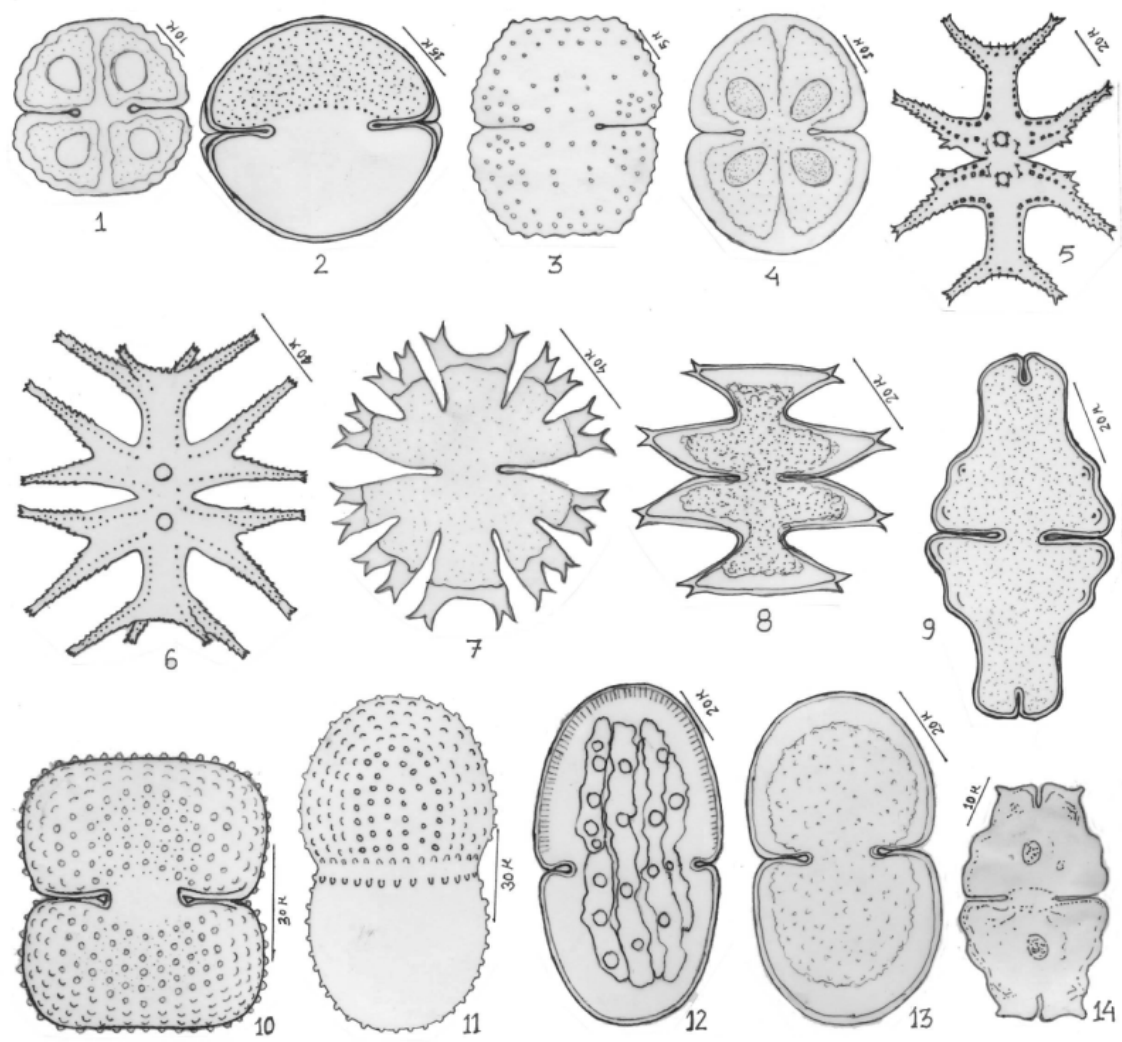

\section{Plate 2}

Fig.1 Cosmarium sublateriundatum West et West, Fig. 2 C. obsoletum (Hantz.) Reinsch, Fig. 3 C. punctulatum Bréb. var. subpunctulatum (Nordst.) Borges, Fig. 4 C. lundellii DelP. var. circulare (Reinsch) Krieg, Fig. 5 Micrasterias tropica Nordst. var. polonica Eichl. et Gutw. f. evoluta Scott et Prescott, Fig. 6 M. mahabuleshwarensis Hobs. var. surculifera Lagerh, Fig. 7 M. radians Turn, Fig. 8 M. pinnatifida (Kütz.) Ralfs, Fig. 9 Euastrum ansatum Ralfs var. dideltiforme Ducell, Fig. 10 Cosmarium margaritatum (Lund.) Roy et Biss. var. sublatum (Nordst.) Krieg, Fig. 11 C. striolatum Näg, Fig. 12 C. maculatiforme Schm, Fig. 13 C. quadratum Ralfs ex Ralfs var. willei (Schm.) Krieg. et Gerl, Fig. 14 Euastrum bidentatum Näg.

33. Remark: Staurastrum cf bifidum Bréb. in Ralfs (Pl. 1, Fig. 21)

Scott, A.M. and G.W. Prescott 1961, P. 86, Pl. 54, Fig. 5
L $37 \mu \mathrm{m}$, W $54 \mu \mathrm{m}$ (with spines), I $16 \mu \mathrm{m}$; CN BH-23; D 25.9.2007

Present specimen has more or less similar dimension with the type but spines are 
S.K.Rai, R.K. Rai and N. Paudel / Our Nature (2008) 6: 58-66

abruptly bent and outer surface distinctly concave.

Distribution: New record for Nepal.

34. Staurastrum javanicum (Nordst.) Turn. var. apiculiferum (Turn.) Krieg. (Pl. 1, Fig. 20)

Scott, A.M. and G.W. Prescott 1961, P. 97, Pl. 44, Fig. 6

L $51 \mu \mathrm{m}$, W $70 \mu \mathrm{m}$, I $14 \mu \mathrm{m}$; CN BH-15; D 24.7.2007

Distribution: New record for Nepal.

35. Staurastrum sexcostatum Bréb. ex Ralfs var. productum (W. West) G.S. West (Pl. 1, Fig. 22)

Kouwets, F.A.C. 1987, P. 251, Pl. 19, Fig. 7 L $42 \mu \mathrm{m}$, W $45 \mu \mathrm{m}$, I $14 \mu \mathrm{m}$; CN BH-1; D 25.6.2007

Distribution: Luitel Bhanjyang, $770 \mathrm{~m}$, Gorkha (Hirano, 1955); Koshi Tappu, 206 m (Rai and Misra, 2008)

36. Desmidium baileyi (Ralfs) Nordst. var. baileyi f. tetragonum Nordst. (Pl. 1, Fig. 16) Nurul Islam, A.K.M. and H.M. Irfanullah 1999, P. 120, Pl. 1, Fig. 1

L $17 \mu \mathrm{m}, \mathrm{W} 24 \mu \mathrm{m}$, I $23 \mu \mathrm{m}$; CN BH-1; D 25.6.2007

Distribution: Koshi Tappu, 206 m (Rai and Misra, 2008)

Conclusion

Present investigation shows that the desmids flora of Bees-hazaar lake is rich and diverse. Among the genera, Cosmarium has the maximum taxa representing by 16 out of 36 whereas Pleurotaenium and Arthrodesmus have monotypes. Here, one specimen (Staurastrum cf bifidum) we have has similar dimensions and morphology of spines with Staurastrum bifidum described by Scott and Prescott (1961) but it has distinctly concave outer surface as in Staurodesmus triangularis (Lagerh.) Teil. described by Kouwets (1987) and abruptly bent down spines. Therefore, it needs further study to be confirmed. For a complete documentation of the specimens, regular seasonal explorations of the lake will be essential.

\section{Acknowledgements}

Authors are grateful to the Chairman, Department of Botany, P.G. Campus, Biratnagar for the laboratory facilities.

\section{References}

Bando, T., T. Nakano and M. Watanabe 1989. The desmid flora of Kathmandu, Nepal. Bull. Natn. Sci. Mus., Ser. B, Tokyo. 15: 1-25.

Bharati, S.G. and G.R. Hegde 1982. Desmids from Karnataka state and Goa, Part III. Genus Cosmarium Corda. Nova Hedwigia 36: 733-757.

Capdevielle, P. and A. Coute' 1980. Quelques Staurastrum Meyen (Chlorophycées, Desmidiacées) rare ou nou veaux pour la France. Nova Hedwigia 33: 859-882.

Flint, E.A. and D.B. Williamson 1998. Desmids (Chlorophyta) in two ponds in Central Canterbury, New Zealand. Algological Studies 91:71-100.

Förster, K 1965. Beitrag zur kenntais der Desmidiaceen-flora von Nepal. Erg. Forschunturn Nepal Himalaya. Khumbu Himal 1, 2: 25-58.

Habib, I. and U.K. Chaturvedi 1995. On some desmids from Nepal. J. Ind. Bot. Soc. 74(14): 277-282.

Habib, I. and U.K. Chaturvedi 1997. Contribution to the knowledge of desmids from Nepal. Phykos 36(1-2): 27-36.

Hickel, B. 1973. Limnological investigations in lakes of Pokhara valley, Nepal. Int. Rev. ges Hydrobiol. 58(5): 659-672.

Hirano, M. 1955. Fresh water algae. In Fauna and flora of Nepal Himalaya (Ed. H. Kihara). Fauna and Flora Research Society, Kyoto, Japan. pp. 542.

Hirano, M. 1963. Fresh water algae from the Nepal Himalaya, collected by a me mber of the Japanese 
S.K.Rai, R.K. Rai and N. Paudel / Our Nature (2008) 6: 58-66

Climbing Expedition. Contr. Biol. Lab., Kyoto Univ., Japan. 16: 1-23.

Hirano, M. 1969. Fresh water algae from Langtang Himal, Nepal Himalaya. Contr. Biol. Lab., Kyoto Univ., Japan. 22: 142.

Hirano, M. 1984. Fresh water algae from east Nepal. Study reported of Baika Junior College 32: 197215.

Ichimura, T. and F. Kasai 1982. New mating groups, group $\mathrm{H}$ and group I of Closterium ehrenbergii Meneghini from Kathmandu valley and terai plains of Nepal. In Reports on the cryptogamic study in Nepal (Ed. Y. Otani). National Science Museum, Tokyo, Japan. pp. 61-73.

Kouwets, F.A.C. 1987. Desmids from the Auvergne (France). Hydrobiol. 146: 193-263.

Kusel-fetzmann, E. 1969. Einige Algen aus Nepal. Khumbu Himal 1(6): 37-56.

Nakanishi, M. 1986. Limnological study in Phewa, Begnas and Rupa lakes. In Studies on distribution, adaptation and evolution of microorganisms in Nepal Himalayas (Ed. Y. Ishida). Ministry of Education, Science and Culture, Kyoto, Japan. pp. 3-13.

Nurul Islam, A.K.M. 1970. Contributions to the knowledge of desmids of East Pakistan, Part I. Nova Hedwigia 20: 903-983.

Nurul Islam, A.K.M. and A.K. Yusuf Haroon 1980. Desmids of Bangladesh. Int. Rev. ges Hydrobiol. 65(4): $551-604$.
Nurul Islam, A.K.M. and H.M. Irfanullah 1999. New records of desmids for Bangladesh- II. Thirteen taxa. Bangladesh J. Bot. 28(2): 117-123.

Prasad, B.N. and P.K. Misra 1992. Fresh water algal flora of Andaman and Nicobar Islands. Vol. II. B. Singh and MP. Singh Publ., Dehradun, India.

Rai, S.K. 2008. Some chlorophycean algae from Maipokhari lake, Ilam. J. Nat. Hist. Mus., Nepal. (Communicated)

Rai, S.K. and P.K. Misra 2008. On some desmids from Koshi Tappu Wildlife Reserve, Nepal. Ecoprint (Communicated)

Scott, A.M. and G.W. Prescott 1961. Indonesian desmids. Hydrobiologia 17(1-2): 1-132.

Shrestha, B. and J.D. Manandhar 1983. Contribution to the algal flora of Kathmandu valley. J. Inst. Sci. Techn. 6: 1-6.

Tiffany, L.H. and M.E. Britton 1952. The algae of Illinois. Hafner Publ. Co., New York. 405p.

Watanabe, M. 1982. Observation on the genus Closterium from Nepal. In Reports on the cryptogamic study in Nepal (Compiled Y. Otani). National Science Museum, Tokyo, Japan. pp. 4759.

Watanabe, M. 1995. Algae from lake Rara and its vicinities, Nepal Himalayas. In Cryptogams of the Himalayas, Vol. 3, Nepal and Pakistan (Eds. M. Watanabe and H. Hagiwara). National Science Museum, Tsukuba, Japan. pp. 1-17. 